\title{
Clinical Characteristics of Anisomyopia in Identical Twins and Management: A Case Report
}

\author{
Viswanathan Sivaraman ${ }^{1}$ and Jothi Balaji ${ }^{1}$ \\ ${ }^{1}$ Affiliation not available
}

March 2, 2022

\section{Introduction:}

Anisomyopia (anisometropic myopia) is defined as an inter-ocular (cycloplegic spherical equivalent) difference greater than one dioptre between eyes among myopic individuals. ${ }^{1,2}$ Though the prevalence of anisomyopia among children is rare, a recent population-based study showed an increased prevalence (5.3\%). ${ }^{3}$ Among Asian young adults and the severity of anisomyopia increased with older age, larger interocular axial length difference and higher cylindrical power. ${ }^{4}$ Often it is associated with amblyopia and aniseikonia (unequal image sizes) and may disrupt the binocular vision. ${ }^{5}$

As both eyes of anisomyopic twins are subjected to similar genetic and environmental conditions, it is difficult to identify the factors responsible for causing the differences in refractive status between eyes. So it is important to understand the clinical characteristics and available interventions to manage the condition. As anisomyopia is not very common, the genetic element of this disorder has not been studied in siblings so far. Herein, I present a case report which elucidates the defining clinical characteristics and management of anisomyopia in identical twins.

The objective of prescribing spectacle correction is correcting the uncorrected refractive error which helps in improving the visual acuity or binocular vision or a combination of both. ${ }^{6}$ To the best of our knowledge very limited guidelines are available prescribing anisometropia using spectacles. Since anisomyopia is very rare this might be the reason for the limited or non-availability of prescribing guidelines. This case report describes how a pair of identical twins with anisomyopia was managed for anisomyopic refractive errors.

\section{Case report}

A pair of 9-year-old identical twin sisters reported to a tertiary eye care centre, visited first in 2017 with a poor distant vision for the last 6 months. No other associated complaints such as headache, deviation of the eyes and there was no history of prior spectacle use. Birth history suggestive of full-term, normal delivery with a low birth weight of $2.00 \mathrm{~kg}$. They were incubated for 10 days. The general health and milestones were normal. Family history of high myopia in father and no history of consanguinity. The refractive error status includes their Cycloplegic (1\% cyclopentolate, $0.5 \%$ tropicamide) refraction, binocular vision status, accommodative response (AR), extraocular movements (EOM), keratometry were assessed and documented (Table 1). AR was measured using an open-field auto refractometer. Both eyes pupils were evaluated using penlight and revealed pupils were regular, round, and reactive to light with no afferent pupillary defect. Visual field was assessed monocularly by confrontation method and it was found to be normal in all four directions in both eyes. There was no abnormality in the anterior segment of either eye and no nystagmus was present. As per the hospital protocol, intraocular pressure was assessed with finger tension and found to be normal in both eyes. Post dilated funduscopic examination with indirect ophthalmoscope with +20.0 D revealed enlarged optic disc with early peripapillary atrophy and normal foveal reflex seen in the right eye and no myopic related fundus changes noted in the left eye. 
Various options of vision correction included single vision glasses, contact lenses for distance were discussed but the parents did not opt for any of those except single vision eyeglasses. So they were prescribed single vision spectacles and were asked to report after 6 weeks for review with new spectacles. The patients visited with the new correction 2 months later, and the best-corrected VA in both eyes were 6/12; N6 (twin1) and 6/9; N6 (twin 2).

Table 1: Visit details of refractive error, binocular vision status and keratometry.

\begin{tabular}{|c|c|c|c|}
\hline & & Twin 1 & Twin 1 \\
\hline & & Right eye & Right eye \\
\hline Baseline Visit ( 2017) & Baseline Visit ( 2017) & Baseline Visit ( 2017) & Baseline Vi \\
\hline UCVA & UCVA & $2 / 60 ; \mathrm{N} 6$ & $2 / 60 ; \mathrm{N} 6$ \\
\hline Cyclo Retinoscopy & Cyclo Retinoscopy & $-8.50 \mathrm{DS} /-1.25 \mathrm{DC} \times 150$ & $-8.50 \mathrm{DS} /-1.2$ \\
\hline Final Rx & Final Rx & $-9.00 \mathrm{DS} /-1.50 \mathrm{DC} \times 150$ & $-9.00 \mathrm{DS} /-1.5$ \\
\hline BCVA & BCVA & $6 / 18 ; \mathrm{N} 6$ & $6 / 18 ; \mathrm{N} 6$ \\
\hline Ocular Motility & Ocular Motility & Full & Full \\
\hline CT - Distance & CT - Distance & Exophoria & Exophoria \\
\hline CT-Near & CT-Near & Orthophoria & Orthophoria \\
\hline $\mathrm{AR}$ & $\mathrm{AR}$ & +1.50 & +1.50 \\
\hline Stereopsis - NV & Stereopsis - NV & 50 arc seconds & 50 arc seconc \\
\hline WFDT - NV & WFDT - NV & Fusion & Fusion \\
\hline $\mathrm{K} 1 / \mathrm{A} 1$ & $\mathrm{~K} 1 / \mathrm{A} 1$ & $47.00 \mathrm{D} / 155$ & $47.00 \mathrm{D} / 155$ \\
\hline $\mathrm{K} 2 / \mathrm{A} 2$ & $\mathrm{~K} 2 / \mathrm{A} 2$ & $48.25 \mathrm{D} / 65$ & $48.25 \mathrm{D} / 65$ \\
\hline Follow up visit 1 (Aug 2018) & Follow up visit 1 (Aug 2018) & Follow up visit 1 (Aug 2018) & Follow up \\
\hline PGP & PGP & $-9.00 \mathrm{DS} /-1.50 \mathrm{DC} \times 150$ & $-9.00 \mathrm{DS} /-1.5$ \\
\hline CVA & CVA & $6 / 9 ; \mathrm{N} 6$ & $6 / 9 ; \mathrm{N} 6$ \\
\hline Cyclo Retinoscopy & Cyclo Retinoscopy & $-8.75 \mathrm{DS} /-0.50 \mathrm{DC} \times 180$ & $-8.75 \mathrm{DS} /-0.5$ \\
\hline Final $R x$ & Final $\mathrm{Rx}$ & $-9.00 \mathrm{DS} /-1.00 \mathrm{DC} \times 150$ & $-9.00 \mathrm{DS} /-1.0$ \\
\hline BCVA & BCVA & $6 / 9 ; \mathrm{N} 6$ & $6 / 9 ; \mathrm{N} 6$ \\
\hline Ocular Motility & Ocular Motility & Full & Full \\
\hline CT - Distance & CT - Distance & Orthophoria & Orthophoria \\
\hline CT - Near & CT - Near & Orthophoria & Orthophoria \\
\hline Stereopsis - NV & Stereopsis - NV & 50 arc seconds & 50 arc seconc \\
\hline WFDT - NV & WFDT - NV & Fusion & Fusion \\
\hline Follow up visit 2 (April 2019) & Follow up visit 2 (April 2019) & Follow up visit 2 (April 2019) & Follow up v \\
\hline PGP & $-9.00 \mathrm{DS} /-1.50 \mathrm{DC} \times 150$ & $-9.00 \mathrm{DS} /-1.50 \mathrm{DC} \times 150$ & $-4.50 \mathrm{DS}$ \\
\hline CVA & $6 / 18 ; \mathrm{N} 6$ & $6 / 18 ; \mathrm{N} 6$ & $6 / 24 ; \mathrm{N} 6$ \\
\hline Cyclo Retinoscopy & $-10.00 \mathrm{DS} /-1.75 \mathrm{DCX} 170$ & $-10.00 \mathrm{DS} /-1.75 \mathrm{DCX} 170$ & $-5.00 \mathrm{DS} /-1.2$ \\
\hline Final $R x$ & -10.00DS/-1.75DCX170 & -10.00DS/-1.75DCX170 & $-5.00 \mathrm{DS} /-1.2$ \\
\hline BCVA & $6 / 9 ; \mathrm{N} 6$ & $6 / 9 ; \mathrm{N} 6$ & $6 / 9 ; \mathrm{N} 6$ \\
\hline Ocular Motility & Full & Full & Full \\
\hline CT - Distance & Orthophoria & Orthophoria & Orthophoria \\
\hline CT - Near & Orthophoria & Orthophoria & Orthophoria \\
\hline Stereopsis - NV & 100 arc seconds & 100 arc seconds & 100 arc seco \\
\hline WFDT - NV & Fusion & Fusion & Fusion \\
\hline \multicolumn{4}{|l|}{ Ocular Biometry } \\
\hline AXL (mm) & 25.47 & 25.47 & 24.04 \\
\hline $\mathrm{ACD}(\mathrm{mm})$ & 3.31 & 3.31 & 3.32 \\
\hline $\mathrm{LT}(\mathrm{mm})$ & 3.32 & 3.32 & 3.34 \\
\hline
\end{tabular}

UCVA: Uncorrected visual acuity, BCVA: Best corrected visual acuity, CT: Cover test, AR: Accommodative 
response, NV: Near Vision, WFDT: Worth four dot test, K1/A1: K-Reading 1/Axis1, K2/A2: K-Reading 2/Axis2, PGP: Present glass power, CVA: Corrected visual acuity, AXL-Axial length, ACD-Anterior chamber depth, LT-Lens thickness

\section{Follow up visit 1}

The patients returned to the clinic for the first follow-up 8 months later with no new complaints. They were using the glasses regularly and had no specific visual complaints. The details of their Cycloplegic refraction and binocular vision status of this visit are given in Table 1. Both the anterior and posterior segment was evaluated and the findings were the same as the last visit. The parents were given various options of vision correction including contact lenses, low dose atropine but the parents did not opt for any of those except eyeglasses. So the patients were prescribed new single vision spectacles and were asked to report after 6 months.

\section{Follow up visit 2}

The patients came for a follow-up visit in early 2019 with no new complaints. Visual acuity status, cycloplegic refraction, and binocular vision status of this visit are given in Table 1. Anterior and posterior segments findings remained unaltered. The baseline documentation of ocular biometry parameters was performed using a non-contact biometer (Argos, Suntech, Inc., Aichi, Japan) and the details are shown in Table 1. The parents were once again given various options of vision correction including contact lenses and low dose atropine but the parents did not opt for any of those except eyeglasses. But this time we insisted on them to consider one of the myopia control strategies as the myopia was progressing in their children. As they were hesitant in considering any of the above options, they were prescribed new single vision spectacles and were asked to report after 6 months.

\section{Follow up visit 3}

The twins returned to the clinic for a follow-up visit in early 2021 with no new complaints. Using the glasses regularly and were able to perform all activities. The details of their Cycloplegic refraction, binocular vision status, and ocular biometry of this visit are given in Table 2. Post dilation, the Funduscopic examination was done and imaged (Figure 1) with a 3Nethra Classic fundus camera (Forus Health, India). The media was clear in both eyes. The cup-to-disc ratio was 0.5 and without any disc edema. However, the right eye optic disc was large in size and vertically oval. Around the optic disc, retinal tessellation and peripapillary chorioretinal atrophy were observed. The left eye revealed normal optic disc size and shape. Both the right and left eye's macular regions didn't show any visible retinal abnormality. For baseline documentation, fundus photography was performed. As per hospital protocol, the kids were re-evaluated by the retina specialist and confirmed that there was no abnormality of optic disc and macula. Since there was a significant increase in the refractive error and corresponding changes in axial lengths, the parents were asked to consider one of the myopia control strategies and this time the parents opted for low dose atropine. So the patients were prescribed low dose atropine eye drops $(0.01 \%)$, new single vision spectacles and were recommended the following lifestyle modifications: 1) to spend more time outdoors (at least 2 hours a day) to take adequate breaks during near work (to follow 20-20-20 rule) 3) to maintain good working distance (not less than 25 cms). The patients were asked to report after 6 months.

Table 2: Final visit details of Refractive error, binocular vision status, ocular biometry.

\begin{tabular}{|c|c|c|c|c|}
\hline Follow up visit 3 & Twin 1 & Twin 1 & Twin 1 & Twin 2 \\
\hline (Apr 2019) & Right eye & Right eye & Left eye & Right eye \\
\hline PGP & $-10.00 \mathrm{DS} /-1.75 \mathrm{DC} \times 170$ & $-10.00 \mathrm{DS} /-1.75 \mathrm{DC} \times 170$ & $-5.00 \mathrm{DS} /-1.25 \mathrm{DCX} 170$ & $-13.00 \mathrm{DS} /-1.25 \mathrm{DC}$ \\
\hline CVA & $6 / 9 ; \mathrm{N} 6$ & $6 / 9 ; \mathrm{N} 6$ & $6 / 60 ; \mathrm{N} 6$ & $6 / 12 ; \mathrm{N} 6$ \\
\hline Cyclo Retinoscopy & $-10.00 \mathrm{DS} /-2.50 \mathrm{DC} \times 180$ & $-10.00 \mathrm{DS} /-2.50 \mathrm{DC} \times 180$ & $-7.00 \mathrm{DS} /-1.75 \mathrm{DCX} 170$ & $-12.50 \mathrm{DS} /-1.50 \mathrm{DC}$ \\
\hline Final $\mathrm{Rx}$ & $-10.00 \mathrm{DS} /-2.25 \mathrm{DC} \times 180$ & $-10.00 \mathrm{DS} /-2.25 \mathrm{DC} \times 180$ & $-7.00 \mathrm{DS} /-1.75 \mathrm{DCX} 170$ & $-12.50 \mathrm{DS} /-1.50 \mathrm{DC}$ \\
\hline BCVA & $6 / 9 ; \mathrm{N} 6$ & $6 / 9 ; \mathrm{N} 6$ & $6 / 9 ; \mathrm{N} 6$ & $6 / 9 ; \mathrm{N} 6$ \\
\hline
\end{tabular}




\begin{tabular}{lllll}
\hline Follow up visit 3 & Twin $\mathbf{1}$ & Twin $\mathbf{1}$ & Twin $\mathbf{1}$ & Twin $\mathbf{2}$ \\
\hline Ocular Motility & Full & Full & Full & Full \\
CT - Distance & Orthophoria & Orthophoria & Orthophoria & Exophoria \\
CT - Near & Orthophoria & Orthophoria & Orthophoria & Exophoria \\
Stereopsis - NV & 50 arc seconds & 50 arc seconds & 50 arc seconds & 200 arc seconds \\
WFDT - NV & Fusion & Fusion & Fusion & Fusion \\
Ocular Biometry & & & & \\
AXL (mm) & 25.85 & 24.98 & 24.98 & 26.93 \\
ACD (mm) & 3.91 & 4.05 & 4.05 & 3.93 \\
LT (mm) & 3.41 & 3.32 & 3.32 & 46.38 \\
K1/A1 & $46.18 \mathrm{D} / 169$ & $46.63 \mathrm{D} / 176$ & $46.63 \mathrm{D} / 176$ & $48.04 \mathrm{D} / 95$ \\
K2/A2 & $48.46 \mathrm{D} / 79$ & $48.15 \mathrm{D} / 86$ & $48.15 \mathrm{D} / 86$ &
\end{tabular}

PGP: Present glass power, CVA: Corrected visual acuity, BCVA: Best corrected visual acuity, CT: Cover test, NV: Near Vision, WFDT: Worth four dot test, AXL-Axial length, ACD-Anterior chamber depth, LT-Lens thickness, K1/A1: K-Reading 1/Axis1, K2/A2: K-Reading 2/Axis2

Figure 1: Posterior pole retinal fundus photography of twin 1, right eye (A): larger and vertically oval optic disc, retinal te

\section{Discussion}

To the best of our knowledge, there is no exclusive prescribing guideline for anisomyopic pediatric cohort as on date. The addressed pediatric anisomyopes spectacle prescription had two challenges. First, the aniseikonic ${ }^{5}$ effect reaches a maximum when it's fully corrected by spectacles, which leads to diplopia or the absence of binocular vision. ${ }^{5}$ Second, pediatric myopia prescribing guidelines recommend optimal or full correction ${ }^{6}$ and it is contraindicated if it's under corrected. The under correction may lead to myopia progression from moderate to high-myopia. High-myopia may cause several other ocular complications including low-vision..$^{7,8}$

Anisomyopia is usually accompanied by strabismus and increases as the degree of anisomyopia increases. ${ }^{9}$ Interesting, the twins had BCVA equal in both the eyes and orthophoric for distance and exophoria for near. The amount of aniseikonia (in percentage) was calculated based on the refractive errors. ${ }^{10}$ In twins 1 and 2, the amount of aniseikonia was $7.9 \%$ and $11.4 \%$ respectively. South $\mathrm{J}$ et al ${ }^{5}$ reported that the amount of aniseikonia greater than $5 \%$ would cause almost no stereopsis, no measurable contrast sensitivity summation and suppression. However, the twin 1 and 2 had normal fusion with stereopsis 50 arc and 200 arcs.

Due to the low socio-economic status and rural background, the twins couldn't afford to go for any other mode of correcting the aniseikonia (Contact lens).

How anisomyopia develops is still unclear and is important to investigate further considering its complications. Premature birth and optical component defects like unilateral cataracts, pseudophakia and vitreous haemorrhage could give rise to a difference in interocular growth. ${ }^{11}$ However, other than refractive errors and premature birth, the twin sisters in this case report had normal eyes, thus the above-mentioned causes are unlikely factors. As the father of the twin sisters had high myopia, the anisomyopia among twin sisters in this case report could be more due to genetic inheritance. ${ }^{11}$

Effective methods are to be implemented to reduce or impede the interocular refractive error difference early in life. In a recent study, Orthokeratology and low dose atropine were compared on anisomyopic children. When followed up after two years, the interocular difference in axial length significantly decreased in the Ortho-k group, whereas it remained stable in atropine groups. Hence it was suggested that Ortho-k might 
be a better choice in reducing the degree of anisomyopia and the progression of myopia. ${ }^{2}$ Depending on how the twin sisters respond to low dose atropine, the Ortho-k or combined treatment strategies could be tried during the subsequent visits, as the twin sisters are at risk of developing further retinal complications due to pathological myopia.

In conclusion, the co-occurrence of anisomyopia in siblings is extremely rare. This case suggests that anisometropic amblyopia if treated early in life could be avoided and phoria status and binocularity could be improved with optimal refractive correction among anisomyopic individuals. When anisomyopia is in progress, the correction shall be aimed to control myopia management than balancing the aniseikonic effect. This report also warrants a need for prescribing guidelines for anisomyopic subjects in different age groups.

Informed Consent: Obtained.

Conflict of Interest: No conflict of interest was declared by the authors.

Financial Disclosure: The authors declared that this study received no financial support.

\section{Reference}

1. Williams K, Hammond C. High myopia and its risks. Community Eye Heal . 2019;32:5-6.

2. Tsai WS, Wang JH, Chiu CJ. A comparative study of orthokeratology and low-dose atropine for the treatment of anisomyopia in children.Sci Rep. 2020;10:1-8.

3. Lee CW, Fang SY, Tsai DC, Huang N, Hsu CC, Chen SY, et al. Prevalence and association of refractive anisometropia with near work habits among young schoolchildren: The evidence from a population-based study. PLoS One . 2017;12(3). e0173519. doi:10.1371/journal.pone.0173519.

4. Samarawickrama C, Mitchell P, Tong L, Gazzard G, Lim L, Wong TY, et al. Myopia-related optic disc and retinal changes in adolescent children from Singapore. Ophthalmology . 2011;118:2050-7.

5. South J, Gao T, Collins A, Turuwhenua J, Robertson K, Black J. Aniseikonia and anisometropia: implications for suppression and amblyopia. Clin Exp Optom . 2019;102:556-65.

6. Wutthiphan S. Guidelines for prescribing optical correction in children. J Med Assoc Thai . 2005: 88:S163-9.

7. Saw SM, Gazzard G, Shin-Yen EC, Chua WH. Myopia and associated pathological complications. Ophthalmic Physiol Opt . 2005;25:381-91.

8. Cho BJ, Shin JY, Yu HG. Complications of pathologic myopia. Eye and Contact Lens. 2016.

9. Barrett BT, Bradley A, Candy TR. The relationship between anisometropia and amblyopia. Prog Retin Eye Res2013;36:120-58.

10. Thill EZ. Theory and Practice of Spectacle Correction of Aniseikonia. In: Clinical Ophthalmology. 1986.

11. Chen J, He JC, Chen Y, Xu J, Wu H, Wang F, et al. Interocular Difference of Peripheral Refraction in Anisomyopic Eyes of Schoolchildren. Narayanan R, editor. PLoS One. 2016 16;11:e0149110. doi:10.1371/journal.pone.0149110 